\title{
Halo Diagnostics Summary
}

Peter Cameron, and Kay Wittenburg

Citation: AIP Conference Proceedings 693, 103 (2003); doi: 10.1063/1.1638332

View online: https://doi.org/10.1063/1.1638332

View Table of Contents: http://aip.scitation.org/toc/apc/693/1

Published by the American Institute of Physics 


\title{
Halo Diagnostics Summary
}

\author{
Peter Cameron $^{1}$ and Kay Wittenburg ${ }^{2}$
}

${ }^{1}$ Brookhaven National Laboraqtory, Upton, NY, USA

${ }^{2}$ Deutsches Elektronen Synchrotron, DESY Germany

\section{INTRODUCTION}

The plenary presentation on Halo Diagnostics ( $T$. Shea) offered the participants several different approaches to organizing their thinking on this diverse and complex subject. In terms of the fundamental goal of understanding halo, the involvement of diagnostics was considered in analytical approaches, in simulations, and in experiments. In terms of the priority given to diagnostics, the range goes from purpose built experiments, to dedicated beam experiments at user facilities, to measurements parasitic to normal operations. In terms of challenges, the task begins with a simple definition of halo, requires clear specification of diagnostics requirements as a variety of next generation machines take shape, confronts the dynamic range and sensitivity problems, and finally addresses two omnipresent challenges to the diagnostics specialist - cost, and building trust in the measurements. With the above considerations in mind, and after a survey of the current state of the art, goals for the workshop were presented.

As the working group efforts got underway, it became clear that even at this workshop a general definition of "Beam Halo" could not be given, because of the very different requirements in different machines, and because of the differing perspectives of instrumentation specialists and accelerator physicists. Definitions were offered both from a geometric perspective (point of departure of beam from Gaussian profile, portion of the density distribution beyond $n$ sigma,...) and from the perspective of the formation mechanism (space charge halo, parametric resonance halo,...). From the Diagnostics point of view, one thing is certainly clear - by definition halo is low density and therefore difficult to measure, due to both low count rates in the tails and the large dynamic range (it is desirable to measure the profile of the core 'simultaneous' with the tails).
At this workshop diagnostics were approximately separated into two subgroups - diagnostics for halo measurement, and diagnostics for halo prevention. Diagnostics for halo measurement presented at this workshop include wire scanners (both solid and laser wires), scrapers, the ionization profile monitor, and (for measurement of longitudinal halo) kickers with gated detectors. Presented diagnostics for halo prevention include electron cloud monitors, instability monitors, the quadrupole moment monitor, energy analyzers, tune monitors, and the $\mathrm{AC}$ dipole. The above two categories are somewhat artificial - it is straightforward to imagine using halo measurement in conjunction with machine tuning as a halo prevention diagnostic, though somewhat more difficult to imagine using the diagnostics in the halo prevention group for halo measurement. In the following, short summaries of the presented devices and their results will be given. The halo measurement devices are summarized in a table.

\section{HALO MEASUREMENT}

Many of the contributions presented refinements to conventional wire scanners to permit tail measurements. Several schemes were described to achieve the required large dynamic range.

In an extracted beamline at the PSR (R. Macek), detection of secondary emission currents from $100 \mu$ $\mathrm{SiC}$ wires led to a dynamic range up to $10^{6}$. The emphasis was on noise suppression, which was achieved by locating the electronics on the beamline, minimizing integration time, limiting bandwidth, performing background subtraction, and auto-zeroing leakage currents and offset voltages. A logarithmic amplifier was used to make possible the required dynamic range with 12 bit digitizers. Measured profiles were approximately Gaussian to almost $10^{-4}$, with broad shoulders beyond that. The demarcation

CP693, Beam Halo Dynamics, Diagnostics, and Collimation, edited by J. Wei, W. Fischer, and P. Manning 
between the central Gaussian and the shoulders was strikingly clean and abrupt. The mechanism of halo production is not understood. Future plans include adding a $-5 \mathrm{KV}$ bias to the wire to minimize electron cloud effects in the measurement.

At LEDA (D. Gilpatrick) a combination of a $33 \mu$ carbon wire for core measurements and a $1.5 \mathrm{~mm}$ thick graphite scraper for the tails are mounted on a single scanning actuator, and make possible a $10^{5}$ dynamic range. Detection was by measurement of secondary emission current for the wire and stopped protons for the scraper. Considerable effort was given to determining optimum bias for both wire $(-12 \mathrm{~V})$ and scraper $(+25 \mathrm{~V})$. Scraper insertion was limited to about $2 \sigma$ by beam heating. The differentiated scraper data was merged with the wire data on-line. Measured profiles appeared to have three distinct regions - a sharp peak extending to $\sim 10^{-1}$, a broader approximately Gaussian shape extending to $10^{-3}$, and broad shoulders beyond that. The mechanism of formation of this structure was not explained.

Both counting and secondary emission techniques were used with good agreement in the slow extracted beam at the AGS (D. Gassner). In the counting measurement, three-fold coincidence horizontal and vertical scintillator telescopes effectively minimized background. Acceptance was about $10^{-4}$ steradian. The scattering targets were $2.5 \mathrm{~mm}$ diameter tungsten rods. Singles peak rates of $\sim 5 \mathrm{M} / \mathrm{sec}$ were a factor of 10 below saturation level. Dynamic range was $10^{-2}$ to $10^{-3}$ for secondary emission and $10^{-3}$ to $10^{-4}$ for counting. The purpose of this installation was to diagnose emittance growth during slow extraction from the AGS. Measurements showed an asymmetric halo. The problem was solved by positioning the extraction kicker and ejection septum further from the beam, causing the slow extracted beam to spend less time in the fringe fields of the AGS main magnets. The wire scanner was then de-commissioned.

Counting techniques were also used with a $7 \mu$ wire at HERA (K. Wittenburg). The very clean background conditions at HERA led to a dynamic range of $>10^{8}$, using the fast scan technique in the beam core and a very slow scan in the beam tail. The efficiency (counts per proton intersecting the wire) was about $10^{-7}$. Measurements clearly showed the effectiveness of the scraper in removing beam tails.

Scintillation counting techniques were supplemented by local silicon detectors as well as the experiment detectors in the HERA-B experiment (K. Wittenburg), which uses an internal target inserted into the halo of the beam. Detection efficiency was (remarkably) greater than 50\%. The large bandwidth of the counting method permitted beam-in-gap measurements between the $\sim 10 \mathrm{MHz}$ bunches. With improved machine performance beam halo practically vanished, a circumstance that made it necessary to artificially generate halo without disturbing the core by means of tune modulation together with beam-beam interaction (C. Montag). With this forced diffusion it was possible to stabilize interactions at the desired rate. It was also shown that the core of the beam and the luminosity at the colliding experiments were not deteriorated by this method, a result of possible significance for the PLL tune measurements mentioned in the next section.

A new readout scheme for wire scanners was successfully tested in Yerevan (reported by $\mathrm{K}$. Wittenburg), where the change of the natural oscillation frequency caused by the heating of the $90 \mu$ beryllium-bronze wire was detected. Temperature resolution of this method is of the order of $10^{-4} \mathrm{C}$. Beams with intensities down to $3.4 \mathrm{nA}$ were successfully scanned, which shows the very high sensitivity of this technique. The authors expect a dynamic range of up to $10^{7}$. Concern was expressed about the effect of ohmic heating of the wire by the beam fields. A vibrating wire scanner has been installed at PETRA at DESY, and is waiting for beam.

In an effort where the focus was not so much on dynamic range as the measurement of fast profile changes, counting techniques were utilized in the fast $(20 \mathrm{~m} / \mathrm{s}) 7 \mu$ carbon flying wire at KEK (S. Igarashi). The flying wire was used to measure the beam profile during injection with the goal of minimizing losses. A series of profiles were acquired by changing the trigger setting in $0.2 \mathrm{~ms}$ increments (each scan needs $4 \mathrm{~ms}$ ). Full beam profiles with good time resolution were assembled by reconstructing the data from successively injected pulses at different sweep trigger settings/wire positions but at the same time after injection. Tune and intensity dependent profile modulations were observed, and were attributed to the space charge driven fourth order resonance. There was good qualitative agreement between measurements and simulations.

Proof-of-principle for a laser wire scanner for the SNS linac was demonstrated at Brookhaven, with further development work and installation taking place at Oak Ridge (S. Assadi). Short ( 10ns) pulses from a $1032 \mathrm{~nm} \mathrm{Nd-YAG} \mathrm{laser} \mathrm{strip} \mathrm{electrons} \mathrm{from} \mathrm{the} \mathrm{H}$ minus beam particles. In the Brookhaven prototype profile measurements were accomplished by using differential current measurements to observe the resulting current notch. The sensitivity of the laser 
wire at Oak Ridge was improved by collecting and measuring the stripped electrons, allowing a dynamic range of up to $10^{4}$. The laser wire method permits measurement of full power beam during normal operations without burning wires, and reduces the danger of expensive contamination of RF cavities in the superconducting portion of the linac.

The RHIC ionization profile monitors ( $R$. Connolly) have been steadily and significantly improved since prototype testing in a transfer line in 1996, and since the acquisition of the first single bunch turn-by-turn injection profiles in the RHIC ring in 1999 (which clearly showed quadrupole oscillations due to injection mismatch). Modifications include improved RF shielding to minimize coupling to beam fields, improved detector geometry to minimize sensitivity to radiation from beam losses, improved geometry of adjacent electric and magnetic fields to prevent migration of non-signal electrons into the detector, improved sweep field geometry and higher sweep voltage to minimize measured profile sensitivity to sweep voltage, and the addition of electron sources to permit calibration. The resulting background reduction has made it possible to begin to probe into the beam tails. A dynamic range approaching $10^{3}$ has been achieved. A similar (but considerably larger) version of this IPM will be built for the SNS Ring.

To meet the stringent $10^{-4}$ loss requirement of the SNS (losses due to beam-in-gap are estimated to be second only to those due to space charge driven halo), longitudinal halo measurement in the SNS Ring (D. Gassner) will be accomplished by a beam-in-gap kicker driving the gap beam onto a scraper, with detection by a fast gated micro-channel plate. In addition, this system will accomplish gap cleaning before extraction, with the scraper retracted and the gap beam landing in the collimators. Without the kicker, the scraper and data acquisition system will also be used for transverse halo measurements.

\begin{tabular}{|c|c|c|c|c|}
\hline Machine & Type & Signal & $\begin{array}{l}\text { Dynamic } \\
\text { range }\end{array}$ & Status \\
\hline $\begin{array}{l}\text { LEDA (LANL) } \\
(6.7 \mathrm{MeV} \text { p) }\end{array}$ & $\begin{array}{l}\text { Scanner+ } \\
\text { Scraper }\end{array}$ & SEM & $10^{5}-10^{6}$ & $\begin{array}{l}\text { Working in control- } \\
\text { system }\end{array}$ \\
\hline $\begin{array}{l}\text { AGS slow extraction line } \\
(2 \mathrm{GeV} \text { p) }\end{array}$ & Scanning Target & $\begin{array}{l}\text { Counting mode } \\
+ \text { SEM }\end{array}$ & $\begin{array}{l}10^{4}-10^{5} \\
10^{2}-10^{3}\end{array}$ & De-commissioned \\
\hline $\begin{array}{l}\text { PSR extraction line (LANL) } \\
(800 \mathrm{MeV} \text { p) }\end{array}$ & $\begin{array}{l}\text { Wire Scanner } \\
\text { with thin wire }\end{array}$ & $\begin{array}{l}\text { SEM } \\
\text { Log amp }\end{array}$ & $10^{6}$ & In regular operation \\
\hline $\begin{array}{l}\text { SNS LINAC } \\
\left(2.5 \mathrm{MeV} \text { to } 1 \mathrm{GeV} \mathrm{H}^{-}\right)\end{array}$ & $\begin{array}{l}\text { Laserwire } \\
\text { scanner }\end{array}$ & $\begin{array}{l}\text { Photo- } \\
\text { neutralization, } \\
\text { electron detection }\end{array}$ & $10^{3}-10^{4}$ & In operation \\
\hline $\begin{array}{l}\text { DESY HERA } \\
(40-920 \mathrm{GeV} \text { p) }\end{array}$ & $\begin{array}{l}\text { Wire Scanner } \\
\text { with thin wire }\end{array}$ & Counting mode & $10^{7}-10^{8}$ & $\begin{array}{l}\text { In operation, } \\
\text { Readout prototype }\end{array}$ \\
\hline $\begin{array}{l}\text { Yerevan }\left(20 \mathrm{MeV} \mathrm{e}^{-}\right) \\
\text {DESY PETRA }(40 \mathrm{GeV} \text { p) }\end{array}$ & $\begin{array}{l}\text { Wire Scanner } \\
\text { with thin wire }\end{array}$ & $\begin{array}{l}\text { Vibrating wire; } \\
\text { natural frequency }\end{array}$ & $10^{6}-10^{7}$ & $\begin{array}{l}\text { Preliminary tests; } \\
\text { More tests planed }\end{array}$ \\
\hline KEK PS (12GeV p) & $\begin{array}{l}\text { Wire Scanner } \\
\text { with thin wire }\end{array}$ & Scintillators & $\sim 10^{3}$ & In operation \\
\hline RHIC (polarized p, ions) & IPM & Current & $10^{2}-10^{3}$ & In operation \\
\hline
\end{tabular}

TABLE 1. Presented instruments for beam profile measurements, their dynamic range and operational status 


\section{HALO PREVENTION}

The summary of the Beam Dynamics working group in these proceedings (A. Fedotov, I. Hoffman) separates halo formation mechanisms into the categories of 'non-resonant' and 'resonant'. The diagnostics presentations for non-resonant mechanisms addressed tools for the observation and analysis of instabilities. The presentations for resonant mechanisms dwelt primarily upon measurement of tune-related parameters.

An overall view of instabilities in both time and frequency domains (M. Blaskiewicz) presented data showing recent measurement of the head-tail instability in RHIC as well as the electron cloud instability in both the AGS Booster and the LANL PSR. The usefulness of principal component analysis in understanding this data was demonstrated. In the effort to understand the conditions for onset of instability, data was presented showing the measurement of longitudinal impedance in RHIC from Schottky spectra. The agreement between theory and measurement in this data was remarkably good. Particularly interesting in this presentation was the observation of long-lived ( $\sim \mathrm{hr}$ ) longitudinal 'hot spots' (solitons?) in the proton beam at store in RHIC, and their possible role in triggering instabilities during acceleration. These hot spots were not observed with gold beams, probably as a result of the damping/diffusion effect of IBS.

The electron cloud instability results from a form of multipacting driven by time gradients of the beam space charge field in the longitudinal tail of the bunch. Different instruments were developed and studied at the PSR (R. Macek) for the measurement of electron cloud parameters. Historically, the presence of electron cloud was observed from pressure rise due to multipacting electrons causing desorption at the beampipe wall, and looking at ion pump currents with sufficient bandwidth provided early time domain information. Early studies of direct electrons utilized simple biased electrodes to collect electrons. Data from these electrons was often puzzling and difficult to interpret, particularly the copious presence of electrons at the wall in bending dipoles in the plane normal to the dipole field (the IPM has clearly demonstrated the practical efficiency of magnetic field in confining electron trajectories). This motivated the development of the Retarding Field Analyzer (RFA), where the presence of a repeller grid permits measurement of the electron energy spectrum at the beampipe wall. The $80 \mathrm{MHz}$ bandwidth of the RFA makes possible detailed study of the time evolution of the electron cloud, and clearly shows the 'trailing edge multipactor' behavior. This multipactor is seeded by electrons released from the beam space charge potential well at the tail of the bunch. A key factor in buildup of the instability is how many seed electrons survive the gap to be captured by the following bunch. To this end, an Electron Sweeping Detector (ESD) was developed. The ESD is basically an RFA with the added capability of applying a fast high voltage $(\sim 1 \mathrm{KV})$ pulse at the beginning of the following bunch. Successful measurements were made of surviving electrons.

In an application of similar techniques to the longitudinal, a high-resolution retarding field energy analyzer (Y. Zou) was developed at UMER (a $10 \mathrm{keV}, 100 \mathrm{~mA}$, electron accelerator) to study the mechanism of beam energy spread and its evolution in the electron beam. Detailed analysis of the device parameters had led to an energy resolution of better than $2 \mathrm{eV}$. First experimental results show excellent agreement between the experiments and the theory (Boersch effect and longitudinal-longitudinal effect). More experiments and further improvements are in preparation.

In the case of resonant mechanisms of halo formation, measurement of tune-related parameters (coherent tune, incoherent tune, beam-beam tune shift, coupling, chromaticity, non-linear tune spread, tune shift due to electron cloud,...) is essential. The application of Phase-locked Loop (PLL) and Schottky techniques to these measurements in RHIC was presented (P. Cameron), as well as plans for utilizing these methods in the SNS Ring. Concern was expressed by Professor Hoffman regarding the reliability of these methods in space-charge dominated beams, and this subject is under investigation. PLL data was also presented showing lock to the large amplitude oscillations of beam in islands of the 2/9 resonance in RHIC, data which might be used in resonance compensation. Preliminary data from a resonant quadrupole monitor was presented, in which the quadruple mode was resonated to improve sensitivity and diminish the dynamic range problem characteristic of this type monitor.

The techniques of electron cooling and stochastic cooling are complementary - electron cooling effectively cools the beam core, whereas stochastic cooling is efficient on the beam tails. In the case of Optical Stochastic Cooling (OSC), undulator magnets would be used for both the pickup and the kicker, and cooling time is power limited rather than bandwidth limited (V. Yakimenko). Present plans on 
the path to eventual OSC in RHIC include summer 2003 testing of an optical parametric amplifier, in which a frequency doubled $\mathrm{CO}_{2}$ laser pumps a nonlinear crystal to amplify the signal (ultimately from the undulator pickup). Gains of over $100 \mathrm{~dB}$ are anticipated. Pickup and kicker should be installed in regions of non-zero dispersion to permit simultaneous longitudinal and transverse cooling. In the case of halo cooling, the power limitation can be overcome by adjusting timing of the pump laser to apply power predominantly to the longitudinal beam tails, reducing the practical cooling time from $\sim 1 \mathrm{hr}$ for the entire beam to $\sim<1 \mathrm{~min}$ for the beam tails. A concern is the requirement for an isochronous lattice between pickup and kicker, the requirement being a few microns $\left(\sim 10^{-14} \mathrm{sec}\right)$ at the 12 micron cooling wavelength.

With the AC Dipole (M. Bai), adiabatic turn on and off of a $1 \mathrm{~m}$ long $0.01 \mathrm{~T}$ kicker driving the beam at a frequency close to the betatron tune permits excitation of large amplitude coherent oscillations without measurable emittance growth. Data was presented showing $2 \mathrm{~mm} \mathrm{p}$-p coherent oscillations in RHIC. Measurements accomplished to date include beta functions, phase advance, and linear coupling. By driving the beam at the spin tune rather than the betatron tune, this kicker is also used as a spin flipper for the polarized beam program. Possible applications of this method to halo measurements are under study.

RHIC boasts an active and well-organized Beam Experiments program focused on improvements in machine performance. Essentially all experiments in this program have direct bearing on halo formation. In a summary of beam experiments results (F. Pilat), data was presented on measurements of dynamic aperture, diffusion coefficients (both at injection and store), beam transfer functions in the $4-8 \mathrm{GHz}$ range (in preparation for initial stochastic cooling efforts in 2004), IR triplet nonlinearities and corrections, beam-beam tune shift vs. crossing angle, nonlinear chromaticity, resonance compensation, and pressure rise/electron cloud. Plans for 2004 include addressing the requirements for luminosity increases and RHIC upgrade plans, improved machine modeling, and increased collaboration with FNAL and CERN.

\section{CONCLUSION}

Wire scanners and scrapers define the state of the art in halo measurements, with high sensitivity and huge dynamic range. With the variable-delay trigger method, and given the possibility of repeated replication of beam conditions, wire scanners can measure fast profile changes. With continuous steady improvements, the IPM is starting to make contributions to halo measurement, including fast profile changes without the requirement of repeated replication of beam conditions. Beyond these breadand-butter tools, there are evolving a variety of nice (and sometimes even fancy) instruments to measure the parameters which may drive beam tails. A difficult challenge posed by the accelerator physicists is the turn-by-turn (or better yet, bunch-by-bunch) measurement of beam parameters, both in static and ramped machines. 